\title{
Estigma social percibido y estrategias de afrontamiento en personas con enfermedad mental
}

Perceived Social Stigma and Coping Mechanisms in People with Mental Illness

\section{Enrique Jesús Sáez Álvarez', María Dolores Burguete Ramos', José Ramón Martínez Riera ${ }^{2}$, Fernando Daniel Moreno Rupérez}

${ }^{1}$ Universidad Católica de Valencia

${ }^{2}$ Universidad de Alicante

Contacto: enriquejesus.saez@ucv.es

Fecha de recepción: 02 de mayo de 2018 / Fecha de aceptación: 12 de junio de 2018

\begin{abstract}
Resumen
En el campo del estigma hacia los pacientes con trastornos mentales, se puede apreciar un cambio en la identidad de la persona, que pasa de una historia previa a ser para los otros "el enfermo", "el esquizofrénico" o "el loco". Se puede estudiar esta nueva identidad desde el punto de vista del paciente, asumiendo que incluye su propia interiorización de la percepción del medio sobre él mismo.

Objetivo: Describir las experiencias de un grupo de sujetos con enfermedades mentales acerca del estigma social percibido y sus estrategias de afrontamiento.

Metodología: Estudio cualitativo, la investigación se desarrolló a través de la técnica de la entrevista individual semiestructurada, individual a sujetos que pertenecían a una Asociación de Familiares y Personas con Enfermedad Mental (AFEMPES); las narraciones se grabaron en audio y se trascribieron posteriormente, realizando un análisis del discurso.

Resultados: Las áreas en que se aprecia un mayor estigma social percibido son aquellas que se relacionan con los problemas de socialización. Los métodos de afrontamiento empleados son la aceptación resignada y el conformismo en la mayoría de los casos.
\end{abstract}

Palabras clave: enfermedad mental, estigma, identidad, familia, relaciones sociales.

\begin{abstract}
In the field of stigma towards patients with mental disorders, we can see a change in the identity of the person, which goes from a previous history to being for the others "the sick person", "the schizophrenic" or "the crazy person". This new identity can be studied from the point of view of the patient, assuming that it includes his own internalization of the perception of the environment about himself.

Objective: To describe the experiences of a group of subjects with mental illnesses about perceived social stigma and their coping strategies.

Methodology: Qualitative study, the research was developed through the technique of semi-structured individual interview, individual to subjects that belonged to an Association of Relatives and People with Mental Illness (AFEMPES); the narrations were recorded in audio and later transcribed, making an analysis of the discourse.

Results: The areas in which there is a greater perceived social stigma are those that are related to socialization problems. The coping methods used are resigned acceptance and conformism in most cases.
\end{abstract}

Keywords: mental illness, stigma, identity, family, social relationships.

\section{Introducción}

El término estigma tiene origen griego y era utilizado para hacer referencia a rasgos físicos que podían considerarse negativos o extraños en el estatus moral de aquel que los presentaba. Tales rasgos podían ser cor- tes, quemaduras... que advertían del estatus de esclavo de la época. Con la llegada del cristianismo, se le añadieron otros significados metafóricos que se referían a los signos del cuerpo provocados por la gracia divina que desembocaban en erupciones cutáneas y, por otro lado, los referentes médicos que provocaba esta alu- 
sión religiosa a los rasgos corporales de perturbación física. Actualmente se mantiene un sentido similar al original, pero haciendo referencia al mal en sí mismo y no al mal como algo negativo físicamente sobre tu cuerpo ${ }^{1}$.

El estigma aplicado a la enfermedad mental se traduce como un fenómeno global que tiene un impacto sobre el desarrollo social/laboral de los pacientes y sobre su uso de los recursos de salud a los que pueden acceder $^{2}$. Como principales resultados negativos del estigma tenemos el ostracismo y aislamiento, la desmoralización, la desesperanza, la baja autoestima y una reducción de la búsqueda de ayuda. A veces estos resultados pueden ser peores que la misma sintomatología ${ }^{3}$.

La identidad es un concepto complejo y multidimensional que incluye aspectos sociales y culturales. En el campo del estigma hacia los pacientes con trastornos mentales, se puede apreciar un cambio en la identidad de la persona, que pasa de una historia previa a ser para los otros "el enfermo", "el esquizofrénico" o "el loco". Se puede estudiar esta nueva identidad desde el punto de vista del paciente, asumiendo que incluye su propia interiorización de la percepción del medio sobre él mismo ${ }^{4}$.

Por otra parte, el término de calidez humana hace referencia al pensar en los demás y en su manera de sentir, para ajustar nuestra conducta y mostrar así amabilidad, comprensión y alta disposición de servicio. La calidez humana es espontánea y desinteresada ${ }^{5}$. Cuando dicha experiencia puede ser atribuida a un grupo con el que se interactúa, podemos hablar de calidez social. En este sentido, desde la psicología positiva, con un enfoque centrado en las virtudes, emociones positivas y fortalezas que, obviamente, influyen en la calidad de vida y en la salud mental y cuyo acercamiento y estudio pueden ayudar a mejorarlas en algunos sujetos y mantenerlas en otros ${ }^{7}$, quizá, ante el término estigma, podría ser interesante comenzar a estudiar este fenómeno desde su contraparte, la calidez social, desde el punto de vista personal de cada individuo, es decir, la calidez social percibida.

\section{Objetivos}

\section{Principal:}

Describir las experiencias de un grupo de sujetos con enfermedades mentales acerca del estigma social percibido y sus estrategias de afrontamiento.

\section{Secundarios:}

- Identificar las áreas en que se aprecia una menor calidez social percibida.

- Enumerar los métodos de afrontamiento empleados por los participantes para desenvolverse en situaciones de baja calidez social percibida

- Examinar el mapa relacional de los sujetos a estudio, con el fin de analizar el grado de influencia en su autopercepción.

\section{Sujetos y método}

En la elaboración del trabajo se utilizó una metodología cualitativa. La investigación se desarrolló a través de la técnica de la entrevista individual semiestructurada. Se establecieron unos objetivos específicos previos a la realización de las entrevistas, por lo que el enfoque seguido ha sido deductivo. Se ha utilizado categorías analíticas para describir y explicar las materias objeto del estudio.

\section{Recogida de la muestra}

El primer paso fue solicitar el permiso de la institución con la que se trabajó para la obtención de la misma; en este caso, se trata de la Asociación de Familiares y Personas con Enfermedad Mental (AFEMPES), la cual se encuentra en Paterna (Valencia). Una vez obtenida, se redactó una hoja de información al participante y el consentimiento informado. Se seleccionaron un grupo de personas con esquizofrenia y trastorno bipolar que acudían a la asociación que, tras explicar el proyecto, se prestaron de manera voluntaria. En principio no se discriminó a ninguna de las personas que acudían al centro, descontando aquellas que no cumplieran los requisitos necesarios para su participación, es decir, comprensión de las preguntas planteadas y capacidad de expresión suficiente para obtener una respuesta significativa. No se hizo distinción entre esquizofrenia y trastorno bipolar. La elección como método de investigación de la entrevista semiestructurada $^{8}$ se debió a que esta nos permite profundizar lo suficiente en temas más personales para los entrevistados y, al mismo tiempo, adentrarnos en las vivencias personales con cierta facilidad y sin necesidad de realizar preguntas demasiado complejas, junto con la cantidad adecuada de cuestiones interesantes, sin saturar la entrevista haciéndola demasiado larga y/o compleja. 


\section{Recogida de información}

Las preguntas fueron redactadas y elaboradas anteriormente, tomando en cuenta la revisión de la literatura consultada, por el equipo de investigación. Se dividieron en epígrafes, que incluían temas tales como la vida cotidiana, sus relaciones sociales, la autopercepción, la medicación, el inicio de su enfermedad, etc.

Las entrevistas se realizaron a solas, con excepción de tres de ellas en las que se decidió unir a una de las voluntarias del centro, ya que los entrevistados se sentían más cómodos sabiendo que una persona conocida y de confianza se encontraba presente en la sala. Las narraciones se grabaron en audio, pero no en vídeo, ya que los participantes no deseaban en su mayoría ser visualizados; la duración de la interacción fue de 15-20 minutos, durante los que se abordaron las preguntas pertinentes. A todos los sujetos, en primer lugar, se les reformulaba la información antes proporcionada, con el fin de clarificar cualquier duda; tras ello se les pedía la firma en la hoja de consentimiento informado y, una vez finalizada la entrevista, se les agradecía su participación y se procedía a cerrar la sesión. Por otra parte, el entrevistador se mantuvo alerta y realizó anotaciones sobre el comportamiento no verbal, evitando intimidar o propiciar restricciones en los testimonios del entrevistado ${ }^{9}$.

La investigación se dio por finalizada cuando se obtuvo saturación de los datos.

\section{Resultados}

En principio, la muestra se compuso por ocho participantes. En uno de los casos, dadas las limitaciones presentadas en comprensión y expresión, se realizó la entrevista, pero no pudo plasmarse como parte de los resultados, contabilizándose en siete el número final de participantes.

La media de edad de los participantes fue de 42 años de edad, siendo el menor de 39 y el mayor de 45 años. En su mayoría no poseían estudios; solo tres de ellos poseían el certificado escolar y uno cursó bachillerato, pero sin llegar a finalizarlo. Todos ellos tenían diagnosticado un trastorno mental grave (esquizofrenia o bipolaridad).

Los resultados expresan las diferentes vivencias con sus respectivos códigos y etiquetas:

\section{Vida cotidiana}

La mayoría de los participantes han presentado dificultades en su socialización y se les han presentado problemas a la hora de mantener sus amistades tras la enfermedad; es por ello que la mayoría de sus relaciones de amistad son con otras personas con enfermedades mentales.

Quedaba con uno, que se llama Ángel David, pero... bebe y... bueno, mi padre me decía: "No salgas con Ángel David, porque sus padres no te dejan que no salgas con él, porque dicen ellos que... que tú les incitas a beber". No es verdad, pero bueno.

(Informante 1).

En cuanto la autopercepción, es destacable el hecho de que, a pesar que objetivamente saben que son como los demás, se sienten inferiores, peores que el resto y sin elección. Sus respuestas rondaban siempre en la inferioridad, aunque se sentían personas normales, ni peores, ni mejores, pero con sus peculiaridades.

No, yo soy bueno, soy como todos, con... con mis cosas, pero yo soy como todos. (Informante 2).

La medicación no supone un gran problema para los entrevistados a la hora de socializar o en cuanto a lo que representa para su imagen. Muchos afirman que no la esconden y que, en general, no notan que la gente reaccione mal ante ellas. También hay casos en los que se apreció una ideación positiva en cuanto a tomarse la medicación, por el hecho de que esta les disminuye los síntomas y ya están habituados a ellas.

No, no, yo me tomo mis pastillas y nadie me dice nada, es la pastillita y ya está, que me controla.

(Informante 2).

Bueno... Si, porque te ven tomado algo... a saber, se preguntan para qué es... yo les digo que es para... para las migrañas o el constipado... Algo que se me ocurra... (Informante 4).

En general, los entrevistados asumen con resignación su situación sin intentar modificarla; en todos los casos destaca el conformismo a la hora de afrontar su enfermedad.

Qué voy a hacer, es lo que me ha tocado, lo he pasado muy mal, pero no se puede hacer más... mmm... (Informante 4). 


\section{Relaciones con los otros}

En general, sus amistades son otras personas con enfermedades mentales. A pesar de haber tenido otras amistades anteriormente, actualmente, la mayoría no afirma mantener ninguna debido a diferentes conflictos del pasado.

Sí, yo solo salgo con la gente, los que estamos aquí, aunque con algunos no me hablo porque no quieren saber nada... por lo que creen que soy malo, sus familias, pero yo no he hecho nada. Antes salía con la gente... gente del barrio, mis amigos, pero después, que eeh... que saben lo mío... ya se han ido yendo todos... (Informante 2 ).

La familia parece ser su principal apoyo en todos los casos, incluso algunos que eran reacios al tratamiento acabaron cediendo. Hay casos en los que la familia ha tenido muchísima importancia en sus relaciones sociales, ya que es la que facilita la introducción.

Sí, sí, es que él trabaja ahí, ¿sabes? Y... pues yo le ayudo, me conocen todos. Él es el arbitro... Yo estoy ahí por él, enchufado. Como soy un nervio que no paro, pues estoy en todo, ayudo a todos en general... y... esas cosas. Cuando les hace falta algo, pues yo voy y lo tengo ahí en un momento. (Informante 3 ).

En cuanto a las relaciones de pareja, no se consideran con posibilidades suficientes como para centrarse en algo así, renuncian a ello o creen que no son capaces.

No, porque... no puedo dar nada bueno a ninguna persona, no puedo dar nada bueno. (Informante 1).

En cuanto a tener relaciones sexuales, el rechazo es la principal respuesta. Las razones rondan entre la incapacidad física, debido a la disfunción eréctil, y de nuevo, que no consideran que pueda existir deseo sexual hacia ellos por parte de otras personas.

Pero síque lo pienso a veces, o sea, decir que quiero una novia, no, pero, claro, uno se acuerda y no sé. Ya lo mío no funciona (haciendo referencia al pene) ni nada (ríe) pero es el cariño. (Informante 2)

\section{Impacto social del diagnóstico}

En lo referente a los posibles acosos que hayan sufrido, suelen coincidir en que desde la infancia hasta la vida adulta han tenido que lidiar con insultos, ofensas. No todos saben diferenciar entre el acoso sufrido en la infancia de forma más habitual entre niños o el referente a temas de salud mental. También es difícil diferenciar entre qué edades. Como métodos de afrontamiento no destacamos ninguno puesto que, en general, no se ha intentado poner remedio a esto; principalmente intentaban evitar el conflicto.

Y yo creo que están solos porque me están haciendo bullying, porque dice, dirán... se alegran de mis... desgracias, se alegran de mis desgracias y se encierran en casa. (Informante 1 ).

A mí sí, mucho, de siempre, pero bueno... ya sabes, desde el colegio... yo era el rarito... con las gafas... se metían conmigo, siempre era yo. De siempre, vamos (Rie). Y ahora porque ya tenemos una edad... (Informante 4).

En general, afirman que son muchas las personas que les han atacado sin razón solo por el hecho de tener una enfermedad mental. Esto provoca un resentimiento en ellos.

Es maldad, en el fondo es maldad lo que tiene la gente. Pero... si dirán eso, dirán: buah, menudo perdido de tío tenemos aquí en España. Lo único.. vago y maleante, vago y maleante. (Informante 1).

En cuanto a informar de su enfermedad, en algunos casos se mostraban más abiertos con la idea de contarlo, pero solo en circunstancias en las que se haya adquirido una mayor confianza con esa nueva persona. Otros sujetos se oponían a exponerse de manera rotunda. Hablaban de la negativa de la familia a compartir con otros el hecho de que tienen una enfermedad, ya que buscan protegerlos de posibles conflictos y sufrimiento debido al estigma de la sociedad.

No, no, no... yo se lo cuento... se lo cuento a los que conozco mas porque me conocen, sabes? A la gente que me conoce se lo cuento, a esa sí. (Informante 2).

Yo no lo escondo, pero mi hermano no quiere que todo el mundo... lo sepa. Es que ahí... en el fútbol, por ejemplo, si me conocen y al final lo saben... o ven algo raro en mí, que dicen: "Bueno, igual... le pasa algo...", pero como voy con mi hermano pues saben que no pasa nada conmigo. (Informante 3) 


\section{Discusión}

Todas las respuestas orientadas hacia el área de la autopercepción parecen indicar una percepción social negativa sobre la realidad última del sí mismo tal y como es vivida por los participantes, concretamente sobre qué tipo de persona sienten que son. Muchas de las respuestas parecían influidas por la idea en general que tiene la sociedad de las enfermedades mentales y por la estigmatización que padecen. Otras provenían de malas experiencias independientes de cada individuo, de los diferentes problemas a los que se han enfrentado a lo largo de su enfermedad y en cómo, en mayor o menor medida, eran ayudados o rechazados. Es por ello que los datos apuntan hacia una visión del sí mismo que se identifica como un reflejo de aquello que percibe la sociedad, asumiendo así una nueva identi$\mathrm{dad}^{4}$. Las personas estudiadas consideran que, incluso antes de interaccionar, la gente ya tiene una idea negativa de ellos, y solo algunos pensaban que tras conocerles esta idea se volvería positiva y apreciarían que no son "unos locos".

Por otro lado, se ha podido constatar, a través de las diferentes líneas argumentales, que la combinación del tratamiento farmacológico con el apoyo, ya sea familiar o de amistad, genera una mejoría percibida de la sintomatología, lo cual se traduce en una mejora en su vida diaria y da lugar a más probabilidades de sentir bienestar, tanto físico como psíquico, social y espiritual, realidad que también se reproduce en otros estu$\operatorname{dios}^{10,11}$.

Qué duda cabe que dicho sentimiento, ya sea de malestar como de bienestar, tiene cierto correlato con la actitud y métodos de afrontamiento que pueda gestionar cada uno de los participantes. En este punto, es muy importante el refuerzo de la autoestima y confianza de cada persona para poder organizar las diferentes sensaciones y poner en orden sus ideas, con el fin de aprender a proyectar bien su motivación hacia la normalización, lo cual se puede operacionalizar mediante el incremento de la psicoeducación tanto a familias como a pacientes ${ }^{12}$.

Resulta patente, en muchos de los casos, la inexistencia de una intención activa de afrontar el problema o sencillamente el escape, negación o intento de evadir el problema hasta el conformismo. Se hablaría de un método de afrontamiento centrado en la defensa de la autoestima, en el que el sujeto no hace acción para intentar resolver el problema, sino que busca equilibrar el duelo y su nueva situación de vida. Cuando un sujeto actúa de esta manera, su método de afrontamiento no resulta eficiente precisamente para el objetivo pretendido de mejora de la autoestima, faltando, por otro lado, ocasionalmente, la motivación suficiente para llevarlos a cabo con la consiguiente resignación ante su situación ${ }^{13}$.

En el momento de inicio de la enfermedad, se ha visto que la principal sensación es de negación y rechazo de la realidad, hasta llegar a un punto de inflexión en el que el paciente es consciente de que el camino adecuado no es ese, siendo activadores importantes para ellos el sufrimiento percibido de sus personas cercanas o de ellos mismos.

Por último, es necesario destacar una limitación importante del presente estudio: la aproximación al universo de estas personas no ha sido más que un apunte, ya que su realidad es demasiado compleja para poder ser comprendida desde una sola investigación, pero, por una parte, las dificultades de acercamiento en profundidad a este colectivo por sus mismas características y, por otra, con una muestra escasa, no nos han permitido quizás por limitaciones de los mismos investigadores profundizar más en su universo, que esperamos poder hacerlo en futuras investigaciones.

\section{Conclusiones}

- Las áreas en que se aprecia un mayor estigma social percibido son aquellas que se relacionan con problemas de socialización, sentimientos de inferioridad, pérdida de amistades anteriores y sentimientos de rechazo sexual.

- Los métodos de afrontamiento empleados por los participantes para desenvolverse en situaciones de baja calidez social percibida son la aceptación resignada y el conformismo.

- El principal apoyo percibido es la familia, y se aprecia gran dificultad para la consecución de pareja.

\section{Referencias bibliográficas}

1. Goffman, Edwing; Estigma: La identidad deteriorada. Buenos Aires: Amorrortu editores. 2006

2. Sing, Lee: The estigma of schizophrenia: a transcultural problem. Curr OpinPsychiatry. 2002;15(1): 37-41.

3. Sirey, Anne; Alexopoulos, George; Perlick, Deborah; Friedman, Steven; Meyers, Barnnett. Percived stigma and patient-rated severity. PsychiatrServ. 2001; 52(12): 1615-20. 
4. Restrepo, Miguel; Mora, Olga Lucia; Rodríguez, Ana Cristina. Voces del estigma. Percepción de estigma en pacientes y familias con enfermedad mental. Universitas Médica. 2007;48(3):208-210.

5. Ceballos, Paula Andrea. Desde los ámbitos de enfermería, analizando el cuidado humanizado. Ciencia y Enfermería. 2010;6(1):3135.

6. Seligman, Martin. The presidents address. Am Psychol. 1999; 54:559-562.

7. Contreras, Francoise; Esguerra, Gustavo. Psicología positiva: una nueva perspectiva en psicología. Revista Diversitas-Perspectivas en Psicología. 2006;2(2):311-319.

8. Ardèvol, Elisenda; Bertrán Marta; Callén Blanca; Pérez Carmen. Etnografía virtualizada: la observación participante y la entrevista semiestructurada. Athenea Digital. Revista de Pensamiento e Investigación Social [Internet]. 2003 [14 sept 2017] Disponible en http://www.uacm. kirj.redalyc.redalyc.org/articulo.oa?id=53700 305

9. Díaz-Blanco, Laura; Torruco-Garcia, Uri; Martínez-Hernandez, Mildred; Varela-Ruiz, Margarita. La entrevista, recurso flexible y dinámico. Investigación en educación médica. 2013;2(7): 162167.

10. Miklowitz, David; George, Elizabeth; Richards, Jeffrey. A ramdomized study of family focoused psychoeducation and pharmacotherapy in the outpatient management of bipolardisorder. Arch Gen Psychiatry. 2003;60(9): 904-912

11. Rea, Margaret; Tompson, Martha; Miklowitz, David. Family-focused treatment versus individual treatment for bipolar disorder: Results of a randomized clinical trial. J Consult Clin Psychol. 2003; 71(3): 482-492.

12. Jones, Steven. Psychoterapy of bipolar disorder: a review. J Affect Disord. 2004; 80(2-3): 101-114.

13. García Laborda, Ana; Rodríguez Rodríguez, Carlos. Afrontamiento familiar ante la enfermedad. Cultura de los cuidados. 2005;18(2): 45-48. 